NASA/TM-2005-213889

\title{
Acoustics of Excited Jets-A Historical Perspective
}

\author{
Clifford A. Brown \\ Glenn Research Center, Cleveland, Ohio
}


Since its founding, NASA has been dedicated to the advancement of aeronautics and space science. The NASA Scientific and Technical Information (STI) Program Office plays a key part in helping NASA maintain this important role.

The NASA STI Program Office is operated by Langley Research Center, the Lead Center for NASA's scientific and technical information. The NASA STI Program Office provides access to the NASA STI Database, the largest collection of aeronautical and space science STI in the world. The Program Office is also NASA's institutional mechanism for disseminating the results of its research and development activities. These results are published by NASA in the NASA STI Report Series, which includes the following report types:

- $\quad$ TECHNICAL PUBLICATION. Reports of completed research or a major significant phase of research that present the results of NASA programs and include extensive data or theoretical analysis. Includes compilations of significant scientific and technical data and information deemed to be of continuing reference value. NASA's counterpart of peerreviewed formal professional papers but has less stringent limitations on manuscript length and extent of graphic presentations.

- TECHNICAL MEMORANDUM. Scientific and technical findings that are preliminary or of specialized interest, e.g., quick release reports, working papers, and bibliographies that contain minimal annotation. Does not contain extensive analysis.

- CONTRACTOR REPORT. Scientific and technical findings by NASA-sponsored contractors and grantees.
- CONFERENCE PUBLICATION. Collected papers from scientific and technical conferences, symposia, seminars, or other meetings sponsored or cosponsored by NASA.

- SPECIAL PUBLICATION. Scientific, technical, or historical information from NASA programs, projects, and missions, often concerned with subjects having substantial public interest.

- TECHNICAL TRANSLATION. Englishlanguage translations of foreign scientific and technical material pertinent to NASA's mission.

Specialized services that complement the STI Program Office's diverse offerings include creating custom thesauri, building customized databases, organizing and publishing research results ... even providing videos.

For more information about the NASA STI Program Office, see the following:

- Access the NASA STI Program Home Page at http://www.sti.nasa.gov

- E-mail your question via the Internet to help@sti.nasa.gov

- Fax your question to the NASA Access Help Desk at 301-621-0134

- Telephone the NASA Access Help Desk at 301-621-0390

- Write to:

NASA Access Help Desk

NASA Center for AeroSpace Information 7121 Standard Drive

Hanover, MD 21076 
NASA/TM-2005-213889

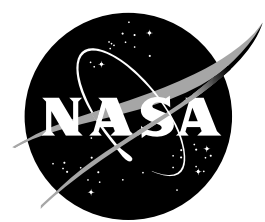

\section{Acoustics of Excited Jets-A Historical Perspective}

\section{Clifford A. Brown}

Glenn Research Center, Cleveland, Ohio

Prepared for the

NOISE-CON 2005

sponsored by the Acoustical Society of America

Minneapolis, Minnesota, October 17-19, 2005

National Aeronautics and

Space Administration

Glenn Research Center 
Available from

NASA Center for Aerospace Information 7121 Standard Drive

Hanover, MD 21076
National Technical Information Service 5285 Port Royal Road Springfield, VA 22100

Available electronically at http://gltrs.grc.nasa.gov 


\title{
Acoustics of Excited Jets-A Historical Perspective
}

\author{
Clifford A. Brown \\ National Aeronautics and Space Administration \\ Glenn Research Center \\ Cleveland, Ohio 44135
}

\begin{abstract}
The idea that a jet may be excited by external forcing is not new. The first published demonstration of a jet responding to external pressure waves occurred in the mid-1800's. It was not, however, until the 1950 's, with the advent of commercial jet aircraft, that interest in the subject greatly increased. Researchers first used excited jets to study the structure of the jet and attempt to determine the nature of the noise sources. The jet actuators of the time limited the range (Reynolds and Mach numbers) of jets that could be excited. As the actuators improved, more realistic jets could be studied. This has led to a better understanding of how jet excitation may be used not only as a research tool to understand the flow properties and noise generation process, but also as a method to control jet noise.
\end{abstract}

\section{Introduction}

The concept that a fluid flow may be influenced by external sources has its origins in the 19th century observations of Leconte (ref. 1), Tyndall (ref. 2), and, later, Rayleigh (ref. 3). However, it was not until the advent of commercial jet engines in the 1950's that others began to further explore these ideas in search of quieter aircrafts and a better understanding of the fluid mechanics in the jet.

The subject of flow excitation covers a vast array of research. As such, the paper presented here is not an exhaustive study of all excited flow research conducted. Rather, this paper seeks to give an overview of the physics of excited jets, primarily in terms of knowledge required to excite a jet. A brief discussion of flow actuators, whose development is crucial if research is to expand to higher Reynolds number jets outside of the laboratory environment, is then addressed. Finally, two research topics, in which jet excitation has played an important role, will also be discussed. The first topic is the application of jet excitation to influence and understand the large-scale coherent structures found in nearly all turbulent flows. While these structures do not directly produce noise (in a subsonic jet), they govern the breakdown of the flow into small-scale turbulence that produces jet noise. Thus, control, exerted in the form of excitation, of the large-scale structures leads to changes in the mixing properties of the jet and, ultimately, the sound produced. The second research topic deals with the direct implications of excitation on the radiated sound. Jets, excited under certain conditions, are observed to produce more broadband noise than in their unexcited state. Excited under other conditions, however, they are observed to produce less broadband noise than in their unexcited state. Following these observations, considerable research has been conducted to determine the mechanism coupling the excitation conditions to the noise produced. The research shows that jet excitation can alter primary properties (turbulent structure, plume mixing rate, and noise produced) of a turbulent shear flow. With a greater understanding of the physics at work and advanced flow, the potential exists for controlled excitation to optimize the properties of a jet for any desired application.

\section{Basic Principles of Jet Excitation}

Jet excitation occurs when a perturbation alters the instabilities already present in the shear layer between the jet and the ambient medium. These perturbations may be created naturally or by intentionally applied forces. While engineers may have little control over the natural perturbations, there are several 
reasons they may want to introduce artificial excitation. As a research tool, jet excitation is used to study flow instabilities, often in pursuit of enhanced mixing for chemical processes, heat transfer, or plume reduction (ref. 4). Artificial excitation has been used to study large-scale coherent structures within the jet plume. In this application, the excitation amplifies the structures of interest and locks them into a regular frequency allowing use of more conventional measurement techniques (ref. 4). Finally, artificial excitation may suppress the turbulence in a free shear flow.

As there are several reasons to introduce excitation to a jet the specific application dictates which instabilities are targeted for excitation. The boundary layer between two fluids with different velocities, temperatures, and densities is inherently unstable. As the fluids mix in search of equilibrium, the boundary layer expands forming Kelvin-Helmholtz instability waves. As the instability waves grow, they break down and dissipate leaving the fluid fully mixed in equilibrium. While the instability waves do not directly radiate sound in a subsonic jet, they govern the formation of the turbulence that does create sound (ref. 5). In a supersonic jet, the instability waves themselves radiate significant sound (ref. 6).

The behavior of the instability waves may be decomposed into modes of oscillation for convenience of analysis. Each mode is characterized by an initial growth (or amplification) rate, governed by linear stability theory and a saturation limit determine by nonlinear effects (ref. 7). Additionally, each mode has a preferred frequency (usually written in nondimensional form as Strouhal number) where maximum amplitude may be obtained. Higher frequency components grow faster and, therefore, saturate closer to the nozzle exit than the lower frequency components that persist further downstream (ref. 7). Within this framework, it has been shown by Plaschko that the thin axisymmetric shear layer near the nozzle exit is unstable to a large number of discrete azimuthal modes (ref. 8). In the fully developed jet downstream of the potential core, however, Batchelor and Gill showed that the jet is only unstable to the first helical mode $(\mathrm{m}=1)$ (ref. 9). Cohen and Wygnanski showed the axial evolution of the lowest seven azimuthal modes $(0 \leq \mathrm{m} \geq 6)$ by calculating the amplification factor at locations from the nozzle exit to four jet diameters downstream in a $\sim 2.7 \times 10^{4}$ Reynolds number jet (ref. 10). It was also observed by Corke, Shakib, and Nagib that while they have similar growth rates, the axisymmetric and first helical modes do not coexist at the same time and place (ref. 11).

Experimentally, many studies have investigated the growth and saturation of instability modes. In a systematic study of jet response to plane wave excitation at several frequencies (axisymmetric mode or $\mathrm{m}=0$ ), Crow and Champagne found that the mode with the maximum attainable amplitude ("preferred mode") at the end of the potential core corresponded to the mode with Strouhal frequency 0.3 (ref. 7). In a later study, Gutmark and Ho showed that the Strouhal frequency of this "preferred mode" varied between experimental jet facilities and concluded that the specific boundary layer characteristics play an important role in the selection of the most amplified mode (ref. 12). Moore excited the axisymmetric mode using acoustic plane waves of different amplitudes. The results show that considerable effects on noise and mixing occur at low levels of excitation if the excitation frequency is near the preferred frequency of the jet (ref. 5). Moore concluded that many possible sources of excitation exist at this level in an actual engine, such as combustion noise. Others have investigated instability growth and saturation in the lowest order helical modes $(\mathrm{m} \leq 2)$ as well as the axisymmetric mode: Chan $(\mathrm{m}=0,1,2)$ (ref. 13), Strange and Crighton $(\mathrm{m}=0,1,2)$ (ref. 14), and Cohen and Wygnanski (ref. 10).

Acoustically, only a small subset of possible instability modes contributes to the sound produced for low Mach number jets. In 1975, Michalke and Fuchs applied Lighthill's analogy to an axisymmetric jet and showed that only the lowest three or four azimuthal modes significantly contribute to the sound field (ref. 15). In later theoretical work, Kopiev and Chernyshev found a similar result by studying vortex ring eigen-oscillations (ref. 16). This theoretical work is supported by experimental observations by Armstrong et al. in the acoustic near field (ref. 17) and by Juve et al. in the acoustic far field (ref. 18).

Most excited jet research has been done in the laboratory on small cold jets with relatively low initial turbulence levels and at relatively low Reynolds number conditions compared to actual engine applications which are hot and have very high Reynolds numbers. A few researchers have investigated the effects of heat and initial turbulence on jet excitability. Lepicovsky, Ahuja, and Salikuddin studied toneexcited heated jets and concluded that the jet sensitivity to excitation is a strong function of jet velocity 
(ref. 19). In general, the researchers found that the addition of heat decreased the excitation level required for a low Mach number jet $(M=0.3)$ to respond while increasing the excitation level needed for a higher Mach number jet to respond $(\mathrm{M}=0.8)$. However, they found no significant change in preferred excitation Stouhal number (ref. 19). Others investigating excited heated jets include Jubelin (ref. 20), Lu (ref. 21), and Vermeulen, Odgers, and Ramesh (ref. 22).

The effect of initial turbulence intensity on jet response to excitation has also been investigated. Raman, Zaman and Rice performed a series of experiments in 1989 where the initial turbulence was varied from 0.15 to 5 percent using screens and boundary layer trips placed inside the jet (ref. 23). The results show that the jet at all initial turbulence levels responded to the excitation; the amplitude required to reach the same level of response, however, increased as the initial turbulence intensity increased. It was also reported that the preferred excitation frequency varied little with changes in turbulence intensity. A similar study, Mankbadi, Raman and Rice, varied only the core turbulence intensity while holding the turbulence intensity in the boundary layer as steady as possible (ref. 24). The researchers found that the jet response was reduced as core turbulence intensity increased. Thus, more turbulent flows require more powerful actuators to excite them. This is a critical issue in developing actuators for practical applications.

\section{Flow Actuators}

Flow actuators generate forces that act on the flow and create the excitation effect. Depending on the actuator, the forces generated may assume different forms. The three common excitation forces are mechanical, pressure (acoustic) waves, and fluidic interactions. Each actuation method carries advantages and disadvantages. Generally, the power, frequency response, and number of actuators must be balanced against the size, weight, and expense of the actuators when selecting an excitation system for a particular application. The following section will discuss physical, pressure, and fluidic actuation methods.

\section{A. Physical Actuators}

Perhaps the simplest excitation method, physical actuators periodically place solid objects in the flow to influence its behavior. This has the effect of redirecting the flow and changing the amplitude of the instability waves. These physical barriers have the advantage of being very strong, able to exert large forces capable of completely redirecting the flow. Frequency response of the actuators, however, is often limited.

A classic example of physical excitation is the use of vibrating ribbons to study Tollmein-Schlichting waves generated during boundary layer transition (ref. 4). Following this idea, Oster and Wygnanski used a thin flap, rotated about one end and driven by two voice coils, where used to study turbulent mixing between two excited streams (ref. 25). Along similar lines, very low Reynolds number bifurcating and blooming jets can be generated by "wobbling the nozzle slightly" (ref. 26).

A modern mechanical actuator combines the forces of physical and fluidic forces. Piezoelectric actuators, like those developed by Wiltse and Glezer (ref. 27), move a small wedge a small distance into the flow. The wedge, which oscillates into and out of the flow, creates fluidic vorticies in its wake. These vorticies generate the actual excitation force. Peizoelectric actuators have been used to study mixing enhancement (refs. 27 and 28) and to excite the smallest scales, within the turbulence dissipation range, in a free shear flow (ref. 29).

\section{B. Acoustic Actuators}

Acoustic waves were the exciting force for the earliest observations of excited flows. In fact, most experimental research on excited flows employed acoustic waves, generated by loudspeakers, as the exciting force. Loudspeakers offer several advantages for use in excited jet research. They are fairly inexpensive and robust. Loudspeakers allow simple control of frequency, relative phase, and amplitude. 
While this makes them a convenient tool, there are limitations on the maximum wave amplitude and frequency that may be generated, restricting the size and speed of the jet that may be influenced. In addition, their size and weight make loudspeakers a poor choice for any application that requires mobility (real-life aircraft engines for example). As rapid mobility is generally not required in a laboratory using small model scale jets, loudspeakers are a good choice. A single loudspeaker, placed in the plenum chamber of a jet rig, is the most common method used for exciting the axisymmetric mode of the jet. Several loudspeakers, mounted at the nozzle exit and set to an appropriate relative phasing, are frequently used to excite the higher modes of the jet.

\section{Fluidic Actuators}

Fluidic actuators assume many forms. From the very simple to the most complex, fluidic actuators function by forcing fluid into the jet plume in the form of pulses or vortices. A simple example, presented by Brown and Ahuja in a 1990 proof of concept paper (ref. 30), consists of a vortex shedding ring placed shortly upstream of the nozzle exit where the fan stream or free stream fluid could generate the exciting vorticies. The frequency of the vortex generation is controlled by the diameter of the rod used to build the shedding ring.

Another actuator, offering more control than a vortex shedding ring, is the "flip-flop" jet or fluidic actuator. The flip-flop jet uses no moving parts but rather relies on the fluid itself to switch paths according to the naturally varying pressure gradient (ref. 31). A feedback tube, whose length and controls the frequency of oscillation (ref. 32), creates regularity in the switching. Flip-flop jets are capable of operating at supersonic exit conditions creating the potential for very large amplitude perturbations (ref. 33). The frequency of oscillation, however, may be a limiting factor (ref. 33). Excitation using flipflop nozzles has been evaluated for use in jet mixing enhancement (ref. 34).

Synthetic, or zero mass jets offer another fluidic excitation device. By varying the pressure in a small orifice, fluid from the flow itself can be periodically sucked in and expelled creating the fluidic pulses that excite the jet (ref. 35). The pressure within the orifice can be controlled using speaker type membranes or by piston actuation. Piston driven synthetic jets have the advantages of higher operating pressures and broader frequency range (ref. 36). Synthetic jets are unique because the net mass flux of the jet is zero.

Spark driven actuators form a final class of fluidic actuators. In this class the exciting force is created by a rapid expansion of fluid due to a combustion process or due to temperature expansion. Combustion driven actuators, investigated by Crittenden, Glezer, Funk, and Parekh in 2001 (ref. 36), burn a gaseous fuel/air mixture in a chamber, expelling the exhaust through an orifice where it acts to excite the flow. The combustion driven actuator has a frequency response limited by the total time required to fill the chamber and burn the contents (which is a function of the chamber size). Another spark driven actuator avoids the problems of combustion by placing a high temperature spark in the directly in the air. The plasma actuator, developed by Samimy et al. (ref. 37), creates a rapid expansion of the air around the nozzle generating the forcing effect. Plasma actuators have good frequency response and have been shown effective on jets up to Mach 1.3 (ref. 38).

Fluidic actuators have, like the flip-flop jet, synthetic jet, and plasma actuator, have developed because of the high amplitude, high frequency forcing required to excite a turbulent high Reynolds number jet. They now allow excitation of jets not possible with the traditional acoustic loudspeaker and, thus, further understanding into the physics of turbulent jets.

\section{Applications}

\section{A. Study of Large-Scale Coherent Structures and Mixing Enhancement}

Coherent structures are generated by flow instabilities and their formation is governed by the initial condition for the flow where the structure originates (ref. 39). While these structures do not directly 
radiate sound, they govern the production of the small-scale turbulence that is responsible for generating sound (ref. 5). To maintain control over a turbulent flow, Crow and Champagne theorized that either the vorticity must be forced directly using body forcing or the boundary conditions must be controlled at all times (ref. 7). As it is not practical to directly force the vorticity everywhere in the flow, the only avenue of control remaining is the boundary conditions. But, if coherent structures are indeed governed by the initial condition, controlling the boundary condition at the nozzle exit is a logical method to influence at least the initial generation of coherent structures. For this reason, control of the jet seems to be limited to the initial region of the jet. Hussain and Zaman noted that no phase-locked coherent structures could be measured beyond 6 jet diameters downstream of the nozzle exit (ref. 40). Additionally, the turbulence intensity in the forced jet trends to the same levels as that in the unforced jet between six and eight jet diameters downstream of the nozzle exit (ref. 7).

Studies dealing with large-scale structures often apply excitation techniques. Although it is possible to measure large-scale structures without excitation ((ref. 41) for example), the addition of excitation serves to amplify the coherent structures above the background turbulence levels and lock them into a steady formation and pairing frequency. Under the influence of excitation, structures are repeatable in time and space allowing for more traditional phase-average measurements (ref. 42).

In addition to numerous studies on subsonic flows ((refs. 7, 42, 43, 44), others), large-scale coherent structures have also been studied using excitation methods in supersonic flows by Lepicovsky et al. (ref. 45). Using axisymmetric excitation in a fully expanded, shock free supersonic jet (Mach 1.37), the researchers found that the relationship between phase velocity and excitation Strouhal frequency follows the same general trends observed in the subsonic jets. In supersonic jets, these large-scale structures do radiate noise directly and, thus, excitation could play a direct role in altering the sound production.

Large-scale coherent structures in turbulent flows are important energy transport mechanism (ref. 39). Ho and Huang found that multiple vortices converge simultaneously, increased the rate of spreading in a mixing layer, under low-level excitation at a subharmonic frequency (ref. 43). Jet plume mixing enhancement, using a single frequency plane wave, has a maximum limit (ref. 46). This finding led to several studies of using excitation at multiple frequencies simultaneously. Higher spreading rates were found when the jet was excited at the fundamental and subharmonic frequencies (ref. 47). Higher spreading rates were also found when the jet was excited in both the axisymmetic and first helical mode simultaneously (ref. 48). Exerting control over the spreading rate significantly changes the production of small-scale turbulence, the noise generation mechanism in a subsonic jet.

\section{B. Broadband Noise Amplification}

In 1975, Bechert and Pfizenmaier presented some interesting acoustic measurements from a jet, excited by a pure tone, showing an amplification of the broadband noise of 6-7 dB (ref. 49). Moore, in 1977, confirmed the phenomenon and further showed that it can be created by a low level excitation (ref. 5). Deneuville and Jacques showed that amplification was also possible when a broadband excitation source was used (ref. 50). Bechert and Pfizenmaier, also in 1977, generated the broadband amplification effect by exciting the first and second azimuthal modes of the jet (ref. 51). In both cases (broadband source or higher mode excitation), the characteristic tones, which accompanied the broadband amplification in previous experiments, were absent. Jubelin considered both hot jets and supersonic jets and showed that both exhibit broadband amplification when excited (ref. 20). Then, in 1980, Kibens revealed an experiment showing a reduction in broadband noise by up to $10 \mathrm{~dB}$ in an excited jet (ref. 52). In 1985, researchers from the Lockheed-Georgia Company examined the jet noise broadband amplification problem using flow visualization (ref. 44), flow measurements (ref. 53) and acoustic measurements (ref. 54). They concluded that the amplified large-scale structures result in increased smallscale turbulence and that small-scale turbulence is responsible for the increased broadband noise (ref. 54). Zaman also examined the problem of broadband amplification and theorized that the forced vortex pairings created by the excitation were responsible for the increased noise (ref. 55). However, neither theory explains the noise reduction observed by Kibens. 
Jet excitation can increase turbulence and enhance mixing. In 1981, Zaman and Hussain showed that certain excitation conditions would suppress turbulence intensities in jet with laminar initial boundary layers (ref. 56). Flow visualization led to the conclusion that the large-scale structures were destroyed by the excitation before they fully developed, reducing the large-scale fluctuations and, therefore, the turbulence intensity. In a follow on study, Hussain and Hasan reported that the jet noise was reduced when the turbulence was suppressed (ref. 57). These results were not repeatable when the initial boundary layer was turbulent. Thus, for very low Reynolds number jets with laminar boundary layers turbulence levels and, as a result broadband noise levels, can be suppressed. It should be noted that, unlike broadband noise amplification, reliable suppression of broadband noise has not observed. This is due, in part, to the fact that most research uses excitation that enhances turbulence structures and increases mixing, both of with tend to produce increased noise.

\section{Conclusions}

Excitation is one of the very few ways to influence a jet after is passes the nozzle exit. The frequency and amplitude of the perturbations placed on the flow dictate, to a large extent, the behavior of the jet as it develops in the first few jet diameters downstream of the nozzle exit. While considerable work has been done in some areas, there are other areas where there is still much to explore. For example, little research exists on the excitation of azimuthal modes above two. Theory predicts that these modes do not efficiently radiate sound. Investigation, using excitation to amplify these modes, could confirm this and offer insights into the mechanisms at work.

The idea that external forcing at particular levels and frequencies can change the dynamics of a jet is powerful. Research has shown how excitation can enhance certain structures within low Reynolds number jets to dramatically change the turbulence and noise characteristics of the plume. Very little of this research, however, studies the highly turbulent, high Reynolds number jets likely found in real-world applications. This is primarily due to the high frequency and amplitude requirements needed to excite a highly turbulent flow. But, actuator technology is progressing and devices like the flip-flop jets, synthetic jets, and plasma actuators are significantly more capable of dealing the higher requirements of turbulent flows than their predecessors where. Armed with these latest flow actuators and the understanding developed studying the low Reynolds number jets, the next development in excitation technology is poised to move it out of the laboratory and toward more everyday applications.

\section{References}

1. J. Leconte, Phil. Mag. 15, 235 (1858).

2. J. Tyndall, Sound (Longmans, London, 1867).

3. Lord Rayleigh, The Theory of Sound, (Macmillan, London, 1896).

4. E.J. Rice and K.B.M.Q. Zaman, "Control of Shear Flows by Artificial Excitation," NASA TM100201, AIAA-87-2722 (1987).

5. C.J. Moore, "The role of shear-layer instability waves in jet exhaust noise," J. Fluid Mech. 80(2), 321-367 (1977).

6. Dennis K. McLaughlin, Gerald L. Morrison, and Timothy R. Troutt, "Experiments on the instability waves in a supersonic jet and their acoustic radiation," J. Fluid Mech. 69(1), 73-95 (1975).

7. S.C. Crow and F.H. Champagne, "Orderly structure in jet turbulence," J. Fluid Mech. 48(3), 547-591 (1971).

8. Peter Plaschko, "Helical instabilities of slowly divergent jets," J. Fluid Mech. 92(2), 209-215 (1979).

9. G.K. Batchelor and A.E. Gill, "Analysis of the stability of axisymmetric jets," J. Fluid Mech. 14, 529-551 (1962).

10. J. Cohen and I. Wygnanski, "The evolution of instabilities in the axisymmetric jet. Part 1 . The linear growth of disturbances near the nozzle," J. Fluid Mech. 176, 191-219 (1987). 
11. T.C. Corke, F. Shakib, and H.M. Nagib, "Mode selection and resonant phase locking in unstable axisymmetric jets," J. Fluid Mech. 223, 253-311 (1991).

12. Ephraim Gutmark and Chih-Ming Ho, "Preferred modes and the spreading rates of jets," Phys. Fluids 26(10), 2932-2938 (1983).

13. Y.Y. Chan, "Wavelike Eddies in a Turbulent Jet," AIAA J. 15(7), 992-1001 (1977).

14. P.J.R. Strange and D.G. Crighton, "Spinning modes in on axisymmetric jets. Part 1," J. Fluid Mech. 134, 231-245 (1983).

15. A. Michalke and H.V. Fuchs, "On turbulence and noise of an axisymmetic shear flow," J. Fluid Mech. 70(1), 179-205 (1975).

16. V.F. Kopiev and S.A. Chernyshev, "Vortex ring eigen-oscillations as a source of sound," J. Fluid Mech. 341, 19-57 (1997).

17. Ralph R. Armstrong, Alfons Michalke, and Helmut V. Fuchs, "Coherent Structures in Jet Turbulence and Noise," AIAA J. 15(7), 1011-1017 (1977).

18. Daniel Juve, Michel Sunyach, and Genevieve Comte-Bellot, "Filtered Azimuthal Correlation in the Acoustic Far Field of a Subsonic Jet," AIAA J. 17(1), 112-113 (1979).

19. J. Lepicovsky, K.K. Ahuja, and M. Salikuddin, "An Experimental Study of tone-Excited Heated Jets," J. Propulsion 2(2), 149-154 (1986).

20. B. Jubelin, "New Experimental Studies on Jet Noise Amplification," AIAA-80-0961 (1980).

21. H.Y. Lu, "Effect of Excitation on Coaxial Jet Noise as Observed by an Elliptic Mirror," AIAA-812044 (1981).

22. P.J. Vermeulen, J. Odgers, and V. Ramesh, "Acoustic Control of Dilution-Air Mixing in a Gas Turbine Combustor," J. of Eng. for Power 104, 844-852 (1982).

23. G. Raman, K.B.M.Q. Zaman, and E.J. Rice, "Initial turbulence effect on jet evolution with and without tonal excitation," Phys. Fluids A 1(7), 1240-1248, (1989).

24. Reda R. Mankbadi, Ganesh Raman, and Edward J. Rice, "Effects of Core Turbulence on Jet Excitability," AIAA-89-0966 (1989).

25. D. Oster and I. Wygnanski, "The forced mixing between parallel streams," J. Fluid Mech. 123, 91130 (1982).

26. W.C. Reynolds, D.E. Parekh, P.J.D. Juvet, and M.J.D. Lee, "Bifurcating and Blooming Jets," Ann. Rev. Fluid Mech. 2003, 295-315 (2003).

27. John M. Wiltse and Ari Glezer, "Manipulation of free shear flows using piezoelectric actuators," J. Fluid Mech. 249, 261-285 (1993).

28. D.E. Parekh, V. Kibens, A. Glezer, J.M. Wiltse, and D.M. Smith, "Innovative jet flow control Mixing enhancement experiments," AIAA-96-0308 (1996).

29. John M. Wiltse and Ari Glezer, "Direct excitation of small-scale motions in free shear flows," Phys. of Fluids 10(8), 2026-2036 (1998).

30. W.H. Brown and K.K. Ahuja, "Jet Mixing Enhancement by Hydrodynamic Excitation," AIAA-904005 (1990).

31. Hermann Viets, “Flip-Flop Jet Nozzle,” AIAA J. 13(10), 1375-1379 (1975).

32. Ganesh Raman, Edward J. Rice, and David M. Cornelius, "Evaluation of Flip-Flop jet Nozzles for Use as Practical Excitation Devices," J. of Fluids Eng. 116, 508-515 (1994).

33. Ganesh Raman, Michael Hailye, and Edward J. Rice, "The Flip Flop Nozzle Extended to Supersonic Flows," AIAA-92-2724 (1992).

34. Ganesh Raman and David Cornelius, "Jet Mixing Control Using Excitation from Miniature Oscillating Jets," AIAA J. 33(2), 365-368 (1995).

35. Ari Glezer and Michael Amitay, "Synthetic Jet," Ann. Rev. of Fluid Mech. 2002, 503-529 (2002).

36. T. Crittenden, A. Glezer, R. Funk, and D. Parekh, "Combustion-Driven Jet Actuators for Flow Control," AIAA-2001-2768 (2001).

37. M. Samimy, I. Adamovich, J.-H. Kim, B. Webb, S. Keshav, and Y. Utkin, "Active Control of High Speed Jets Using Localized Arc Filament Plasma Actuators," AIAA-2004-2130 (2004). 
38. M. Samimy, J.-H. Kim, I. Adamovich, Y. Utkin, and S. Keshav, "High Speed Jet Control Using Plasma Actuators," To be presented at the 4th International Symposium on Turbulence and Shear Flow Phenomena, Williamsburg, VA., U.S. , June 27-29, 2005.

39. A.K.M.F. Hussain, "Coherent structures - reality and myth," Phys. Fluids 26(10), 2816-2850 (1983).

40. A.K.M.F. Hussain and K.B.M.Q., "The 'preferred mode' of the axisymmetric jet," J. Fluid Mech. 110, 39-71 (1981).

41. F.K. Browand and P.D. Weidman, "Large scales in the developing mixing layer," J. Fluid Mech. 76, 127-144 (1976).

42. K.B.M.Q. Zaman and A.K.M.F. Hussain, "Vortex pairing in a circular jet under controlled excitation. Part 1. General jet response," J. Fluid Mech. 101(3), 449-491 (1980).

43. Chih-Ming Ho and Lein-Saing Huang, "Subharmonics and vortex merging in mixing layers," J. Fluid Mech. 119, 443-473 (1982).

44. K.K. Ahuja and M.C. Whiffen, "Tone Excited Jets, Part II: Flow Visualization," J. Sound Vib. 102(1), 63-69 (1985).

45. J. Lepicovsky, K.K. Ahuja, W.H. Brown, and R.H. Burrin, "Coherent Large-Scale Structures in High Reynolds Number Supersonic Jets," NASA CR-3952 (1985).

46. Ganesh Raman, Edward J. Rice, and Reda R. Mankbadi, "Saturation and the Limit of Jet Mixing Enhancement by Single Plane Wave Excitation: Experiment and Theory," AIAA-1988-3613 (1988).

47. Ganesh Raman and Edward J. Rice, "Subharmonic and Fundamental High Amplitude Excitation of an Axisymmetric Jet," AIAA-89-0993 (1989).

48. Ganesh Raman, Edward J. Rice, and Eli Reshotko, "Naturally Occurring and Forced Azimuthal Modes in a Turbulent Jet," NASA TM-103692 (1991).

49. D. Bechert and E. Pfizenmaier, "On the Amplification of Broad Band Jet Noise by a Pure Tone Excitation," J. Sound Vib. 43, 581-587 (1975).

50. P. Deneuville and J. Jacques, "Jet Noise Amplification: A Practically Important Problem," AIAA77-1368 (1977).

51. Dietrich W. Bechert and Eberhard Pfizenmaier, "Amplification of Jet Noise by Higher-Mode Acoustical Excitation,” AIAA J. 15(9), 1268-1271 (1977).

52. Valdis Kibens, "Discrete Noise Spectrum Generated by an Acoustically Excited Jet," AIAA J. 18(4), 434-441 (1980).

53. J. Lepicovsky, K.K. Ahuja, and R.H. Burrin, “Tone Excited Jets, Part III: Flow Measurements,” J. Sound Vib. 102(1), 71-91 (1985).

54. K.K. Ahuja and D.F. Blakney, “Tone Excited Jets, Part IV: Acoustic Measurements," J. Sound Vib. 102(1), 93-117 (1985).

55. K.B.M.Q. Zaman, "Far-field noise of a subsonic jet under controlled excitation," J. Fluid Mech. 152, 83-111 (1985).

56. K.B.M.Q. Zaman and A.K.M.F. Hussain, "Turbulence suppression in free shear flows by controlled excitation," J. Fluid Mech. 103, 133-159 (1981).

57. A.K.M.F. Hussain and M.A.Z. Hasan, "Turbulence suppression in free turbulent shear flows under controlled excitation. Part 2. Jet-noise reduction,” J. Fluid Mech. 150, 159-168, (1985). 


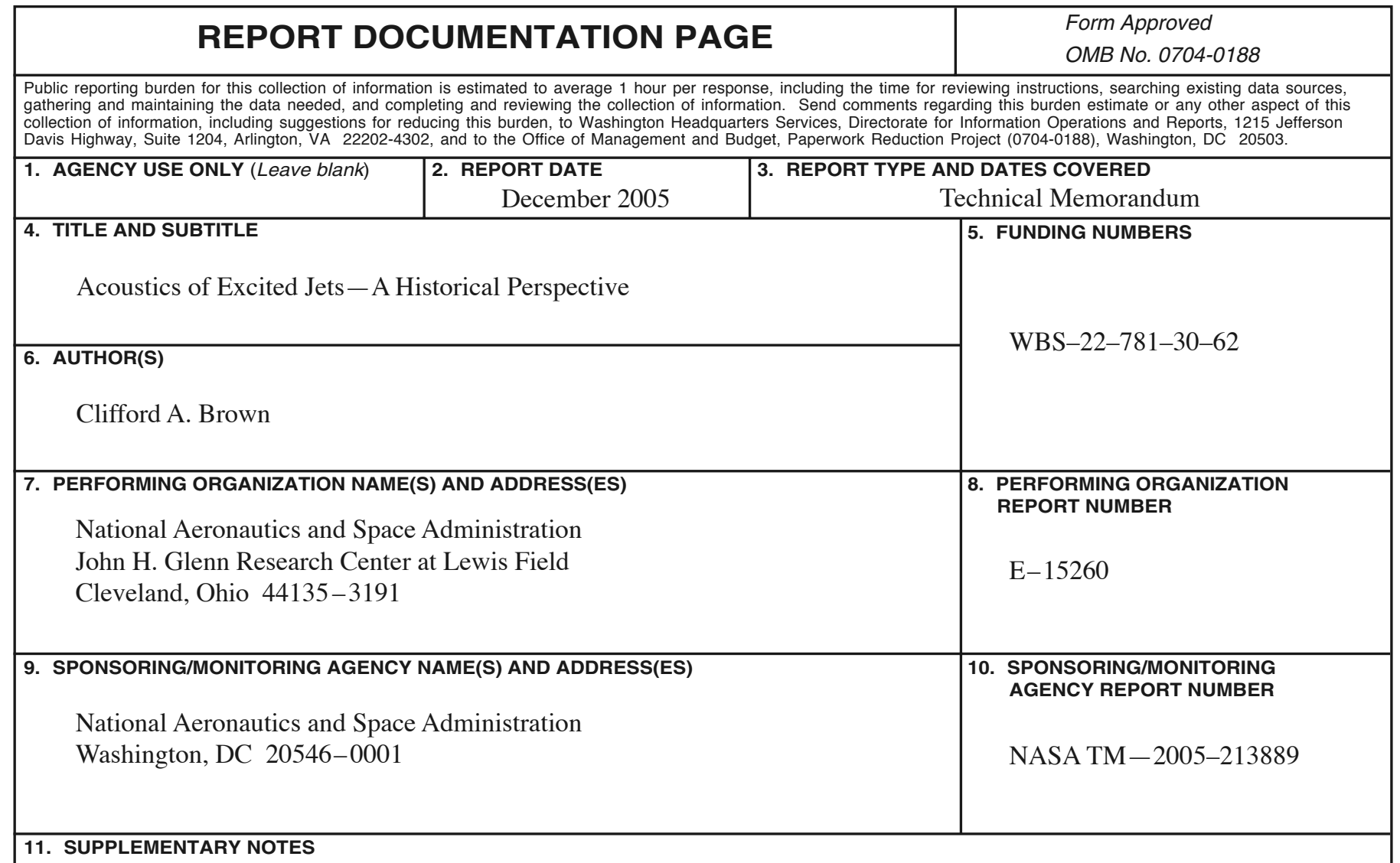

Prepared for NOISE-CON 2005 sponsored by the Acoustical Society of America, Minneapolis, Minnesota, October 17-19, 2005. Responsible person, Clifford A. Brown, organization code RTA, 216-433-2896.

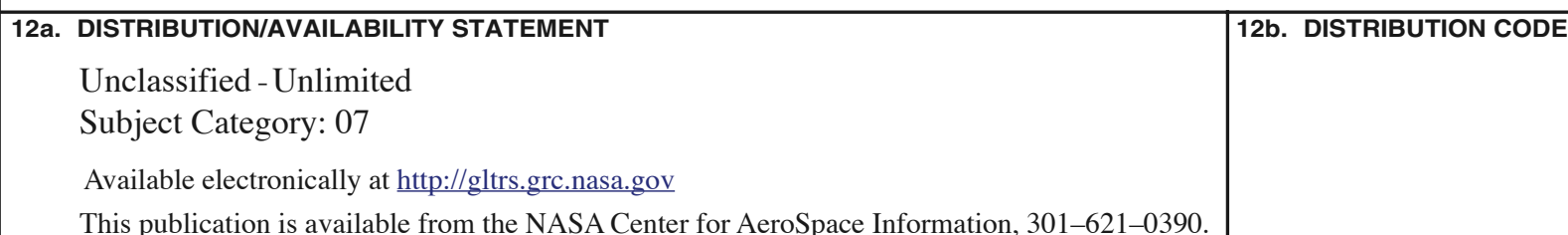

\section{ABSTRACT (Maximum 200 words)}

The idea that a jet may be excited by external forcing is not new. The first published demonstration of a jet responding to external pressure waves occurred in the mid-1800's. It was not, however, until the 1950's, with the advent of commercial jet aircraft, that interest in the subject greatly increased. Researchers first used excited jets to study the structure of the jet and attempt to determine the nature of the noise sources. The jet actuators of the time limited the range (Reynolds and Mach numbers) of jets that could be excited. As the actuators improved, more realistic jets could be studied. This has led to a better understanding of how jet excitation may be used not only as a research tool to understand the flow properties and noise generation process, but also as a method to control jet noise.

\begin{tabular}{|c|c|c|c|}
\hline 14. SUBJECT TERMS & & & $\begin{array}{c}\text { 15. NUMBER OF PAGES } \\
14\end{array}$ \\
\hline N 7540-01 & & & $\begin{array}{l}\text { ndard Form } 298 \text { (Rev. 2-89) } \\
\text { cribed by ANSI Std. Z39-18 } \\
102\end{array}$ \\
\hline
\end{tabular}



